\title{
PARTICULARITIES OF THE INTESTINAL MICROBIOME COMPOSITION IN ROMANIAN PATIENTS WITH METABOLIC SYNDROME AND TYPE 2 DIABETES
}

\author{
Raluca A. CORB ARON ${ }^{1}$, Delia M. TIT ${ }^{1}$, Radu MOLERIU ${ }^{2}$, Cosmin M. VESA ${ }^{1}$, \\ Sergiu BUNGAU ${ }^{1}$, Alexandra G. TARCE ${ }^{1}$, Tapan BEHL ${ }^{3}$, Simona G. BUNGAU ${ }^{\star} \bowtie$ \\ ${ }^{1}$ Faculty of Medicine and Pharmacy, University of Oradea, Oradea, Romania \\ ${ }^{2}$ Faculty of Mathematics and Computer Science, West University of Timisoara, Timisoara, Romania \\ ${ }^{3}$ Chitkara College of Pharmacy, Chitkara University, Punjab, India
}

Received 04 Nov 2021, Accepted 28 Nov 2021

https://doi.org/10.31688/ABMU.2021.56.4.04

\begin{abstract}
Introduction. The composition of gut microbiota can be affected by type 2 diabetes mellitus (T2DM) and metabolic syndrome (MS), its improvement is frequently ignored in metabolic disorders.

The objective of the study was a comparative evaluation of the faecal microbiota of healthy controls and of T2DM and MS patients $(n=150)$.

Materials and methods. A retrospective crosssectional study was performed, between July 2019. December 2020 in the Bio-standard laboratory Oradea, Romania. A group of 75 patients with non-insulin dependent T2DM and MS (study group $=$ SG) and 75 healthy subjects (control group $=\mathrm{CG}$ ) has been included in the study. The composition of the microbiome and the influence of oral antidiabetic treatment on the microbiome in SG patients were compared.
\end{abstract}

Results. 8 out of 19 species of the analysed bacteria had a different relative abundance in the SG, compared to CG; Enterococcus spp. were significantly higher (3.18x106 vs $1.94 \times 106, p=0.004)$, while Akkermansia

\section{RÉsumé}

Particularités de la composition du microbiome intestinal chez les patients roumains atteints de syndrome métabolique et de diabète de type 2

Introduction. La composition du microbiote intestinal peut être affectée par le diabète de type 2 (T2DM) et le syndrome métabolique (SM), son amélioration est fréquemment ignorée dans les troubles métaboliques. L'objectif de l'étude était une évaluation comparative du microbiote fécal de témoins sains et de patients DT2 et SM $(n=150)$.

Matériaux et méthodes. Une étude transversale rétrospective a été réalisée entre juillet 2019 et décembre 2020 dans le laboratoire Bio-standard Oradea, Roumanie. Un nombre de 75 patients atteints de DT2 non insulinodépendant et de SM (groupe d'étude = $\mathrm{GE}$ ) et 75 sujets sains (groupe témoin $=\mathrm{GT}$ ) ont été inclus dans l'étude. La composition du microbiome et l'influence du traitement antidiabétique oral sur le microbiome chez les patients SG ont été comparées. 
muciniphila $(2.55 \times 109$ vs. $4.66 \times 109, \mathrm{p}<0.001)$ and Eubacterium spp. (3.51x108 vs 4.16x108, p=0.047) had significantly lower values in the SG vs. CG.

Conclusions. The results indicated differences in the relative abundance of microbial species between the two groups, with significant changes in gut microbiome in the SG.

Keywords: metabolic syndrome, type 2 diabetes, weight status, glucidic profile, lipidic profile.

\author{
List of abbreviations: \\ BMI - body mass index \\ CG - Control group \\ DBP - diastolic blood pressure \\ HbA1c - glycosylated haemoglobin A1c \\ HDLc - high-density lipoprotein cholesterol \\ LDLc - low-density lipoprotein cholesterol \\ MS - metabolic syndrome \\ NAFLD - non-alcoholic fatty liver disease \\ SCFA- short-chain fatty acid \\ SG - study group \\ SBP - systolic blood pressure \\ T2DM - type 2 diabetes
}

\section{INTRODUCTION}

The most common metabolic disorder worldwide is type 2 diabetes mellitus (T2DM), its specific features including peripheral insulin resistance in the liver, skeletal muscle and adipose tissue, and deficiencies in the insulin secretion ${ }^{1}$. In several developed countries of North America and Europe, the prevalence of T2DM grew alarmingly over the last decades ${ }^{2}$. An increased incidence of obesity and metabolic syndrome (MS) (two factors strongly associated with the risk of diabetes) was also observed ${ }^{3}$. Obesity is linked to hypertension, dyslipidaemia, insulin resistance and hyperglycaemia, these together being known as "metabolic syndrome".

MS has a multifactorial aetiology, with diverse associations between factors like genetic predisposition, behaviours, diet, and the environment ${ }^{5}$. Physiologic risk factors (including lipo-toxicity, cortisol, systemic inflammation, oxidation) are linked to the pathogenesis and appearance of metabolic disorders (i.e. T2DM, obesity, hypertension, non-alcoholic fatty liver disease (NAFLD), etc $)^{6}$. The risk of occurrence for T2DM, as well as of cardiovascular disorders, is enhanced by these metabolic disorders, which also determine increased rates of mortality and morbidity ${ }^{4}$.

Besides genetic background which determines T2DM, other factors like physical activity and diet are also important in the occurrence and gravity of
Résultats. 8 des 19 espèces de bactéries analysées avaient une abondance relative différente dans le GE, par rapport au GT; Enterococcus spp. étaient significativement plus élevés $(3,18 \times 106$ contre $1,94 \times 106, \mathrm{p}=0,004)$, tandis qu'Akkermansia muciniphila $(2,55 \times 109$ contre 4,66x109, p<0,001) et Eubacterium spp. (3,51x108 vs $4,16 \times 108, p=0,047)$ avaient des valeurs significativement plus faibles dans le SG vs. CG.

Conclusion. Les résultats ont indiqué des différences dans l'abondance relative des espèces microbiennes entre les deux groupes, avec des changements significatifs dans le microbiome intestinal dans le GE.

Mots-clés: syndrome métabolique, diabète de type 2 , statut pondéral, profil glucidique, profil lipidique.

T2DM and MS. The advanced knowledge revealed microbiota to be an important factor for human health, presenting new pathways for fundamental and clinical studies of T2DM?

Microbiota consists of various living microorganisms like archaea, bacteria and fungi found in a specific environment ${ }^{8}$. It can regulate health, nutrition and diseases of the host and can be found on or within the host ${ }^{9}$. Although the exact composition of intestinal microbiota is not known, the improvement in metagenomic techniques recently began to reveal the variety of our microbial partners (human microbiome). Each human race contains at least 160 of these species, from a consortium of 1000 to 1150 prevalent bacterial species. $90 \%$ of the bacterial phylotype belongs to the two phyla viz bacteria, followed by Actinobacteria and Proteobacterium, Bacteroidetes and Firmicutes ${ }^{10}$.

The diversity and composition of the gut bacteria have been intensely studied, as well as their impact on health and diseases ${ }^{11}$, including obesity ${ }^{12}$, inflammation ${ }^{13}$, and $\mathrm{T}^{2} \mathrm{DM}^{14}$. Various studies revealed that decreased ratio of Bacteroidetes and increased ratio of Firmicutes were linked to insulin resistance and obesity ${ }^{15,16}$. Contradictory results were revealed by Larsen et al., suggesting that the proportion of Bacteroidetes to Firmicutes was positively and considerably associated with plasma glucose levels, and the Betaproteobacteria class was greatly enhanced in the 
gut microbiome of diabetic subjects ${ }^{17}$. Further research on the connection between metabolic diseases and gut microbiota may determine better therapy schemes, precise monitorization of the disease, as well as new medicines production. In this study, the faecal microbiota of patients with T2DM and MS vs. healthy controls were compared, totalling the information of 150 subjects.

The OBJective OF THE STUDY was to characterize the quantitative composition of intestinal microbial communities in adults with T2DM and MS. Furthermore, the hypothesis that intestinal microbial communities in patients with diabetes are different in individuals treated with oral antidiabetics and hypoglycaemic diet compared to those on a hypoglycaemic diet only was examined. This approach allows the evaluation of the microbiota response in humans, correlated with both drug and food interventions.

\section{Materials ANd methods}

A retrospective cross-sectional study was performed on the database of "Biostandard SRL" laboratories, Oradea, Romania, in the interval July 2019 - December 2020 (18 months), the only medical analysis centre that performed the microbiome analysis in Romania, at that time. The study group (SG) included 75 patients with non-insulin dependent T2DM and with MS. From the same database as well, 75 healthy patients (control group, CG) have been selected. The composition of the microbiome in the two groups was comparatively analysed, and in the SG, the influence of the treatment with oral antidiabetics on the microbiome has been also analysed.

Bacterial groups in faecal samples were quantified by polymerase chain reaction (qPCR), using the RealTimePCR Equipment (DNA Technology, Research and Production" LLC, JSC 2017, Russia), Multiplex kits (Immunodiagnostik Gmbh Germany), MutaPLEX AKM/FEAB PCR and MutaPLEX EU/ BAC/BIF PCR tests, applied after a prior extraction of the genetic material (using ZymoBIOMICS DNA extraction Kit) and spotlighting the an-aerobic groups ${ }^{18}$. Aerobics group and fungus were cultured on convectional culture medias Hektoen, Mackonkey, Columbia Blood Agar, Sabouraud, and quantified by colonies counting technique.

The research was conducted in accordance with the WMA Ethical Declaration of Helsinki ${ }^{19}$, and was approved by the Ethics Commission of the Council of Medicine and Pharmacy Faculty, University of Oradea, Romania (11/27.03.2021). Each patient included in this study signed an informed consent.
The software packages Jasp, SPSS v17 and Microsoft Excel have been used for the statistical analysis. The first step was to obtain a complete descriptive statistic which was performed by calculating the central tendency and descriptive indicators, and by plotting the most important results. After that, the Shapiro - Wilk test was applied to identify data distribution. To see if the observed differences can be considered statistically significant, a Mann - Whitney test (between the two studied groups) was applied. In the end of the study, a risk analysis was run to see if not going under treatment can be considered a risk factor; in this regard, the odds ratio (OR) parameter has been calculated, the $95 \%$ confidence interval was considered, and the chi square test was applied. For all results, $\alpha=0.05$ has been considered.

\section{Results}

\section{Demographic data and clinical characteristics}

Because in most cases it was revealed that the data are not normally distributed $(\mathrm{p}<0.05)$, non-parametrical tests have been chosen. Tests were performed to identify statistical differences between the mean values of age, body mass index (BMI), systolic blood pressure (SBP), diastolic blood pressure (DBP), glycated haemoglobin (HbA1c), glucose of the two groups (with and without disease). Mann - Whitney test has been applied in this purpose and significant differences $(\mathrm{p}<0.05)$ resulted in the case of BMI, HbAlc and glucose (Table 1).

\section{Comparison of the intestinal microbiota}

At the level of intestinal microbiota, 11 bacterial species have been found at identical or very similar levels in both the SG and CG. For Proteus spp., Serratia spp., Morganella morganii, and Pseudomonas spp., the value $1 \times 10^{3}$ was recorded in all cases; for Staphylococcus aureus and Geotrichum spp. 1x102, and Candida Albicans, Candida Nonalbicans, the value 1 x10 was recorded in all cases. Enterobacter spp., Citrobacter spp., and Firmicutes spp./Bacteroides spp. were slightly different in the two groups (Table 2).

The Mann - Whitney test was applied to see if there are significant differences in the number of bacterial species in patients with or without the disease. We obtained significant difference: in Enterococcus spp. $(\mathrm{p}=0.004)$, in Akkermansia muciniphila $\mathrm{p}<0.001$, and in Eubacterium spp. (Table 3). In the SG, Escherichia coli values, Bacteroides and Enterococcus spp., were higher than in the CG, while the rest of the analysed bacteria had lower values in the SG, as Figure 1 reveals.

In the SG, over $50 \%$ of patients did not follow antidiabetic treatment $(54.70 \%)$, following only a hypoglycaemic diet. However, the most used oral 
Table 1. The central tendency and dispersion indicators for age, BMI, glucose level, HbAlc, SBP, DBP

\begin{tabular}{|c|c|c|c|c|c|c|c|c|c|c|c|c|}
\hline \multirow{2}{*}{$\begin{array}{l}\text { Descriptive } \\
\text { statistics }\end{array}$} & \multicolumn{2}{|c|}{ Age } & \multicolumn{2}{|c|}{ BMI } & \multicolumn{2}{|c|}{ Glucose } & \multicolumn{2}{|c|}{ HbAlc } & \multicolumn{2}{|c|}{ SBP } & \multicolumn{2}{|c|}{ DBP } \\
\hline & CG & SG & $\mathrm{CG}$ & SG & $\mathrm{CG}$ & SG & CG & SG & CG & SG & CG & SG \\
\hline Valid & 75 & 75 & 75 & 75 & 75 & 75 & 75 & 75 & 75 & 75 & 75 & 75 \\
\hline Missing & 0 & 0 & 0 & 0 & 0 & 0 & 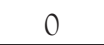 & 0 & 0 & 0 & 0 & 0 \\
\hline Mean & 47.533 & 54.68 & 29.615 & 30.349 & 117.96 & 123.267 & 6.917 & 7.207 & 138.867 & 139.333 & 79.96 & 79.253 \\
\hline $\begin{array}{c}\text { Std. Error o } \\
\text { Mean }\end{array}$ & 0.634 & 1.207 & 0.317 & 0.319 & 2.036 & 2.514 & 0.071 & 0.069 & .786 & 813 & 0.678 & 0.687 \\
\hline Median & 48 & 54 & 29 & 29.69 & 115 & 123 & 6.8 & 7.2 & 140 & 140 & 80 & 80 \\
\hline Mode & 45 & 45 & 27.09 & 29.3 & 132 & 155 & 6.5 & 6.8 & 140 & 140 & 80 & 80 \\
\hline Std. Deviation & 5.49 & 10.45 & 2.749 & 2.766 & 17.636 & 21.775 & 0.618 & 0.595 & 6.807 & 7.039 & 5.874 & 5.953 \\
\hline Variance & 30.144 & 109.194 & 7.557 & 7.653 & 311.039 & 474.171 & 0.382 & 0.354 & 46.333 & 49.55 & 34.498 & 35.435 \\
\hline Skewness & -0.38 & 0.65 & 1.683 & 1.694 & 0.204 & 0.248 & 0.381 & 0.429 & 0.127 & 0.362 & -0.352 & -0.226 \\
\hline Kurtosis & 0.415 & 0.129 & 3.434 & 3.604 & -1.192 & -1.185 & -0.074 & -0.145 & -0.926 & -0.428 & -1.04 & -1.095 \\
\hline $\begin{array}{c}\begin{array}{l}\text { Shapiro-Wilk } \\
\text { (SW) }\end{array} \\
\end{array}$ & 0.968 & 0.964 & 0.846 & 0.838 & 0.946 & 0.937 & 0.979 & 0.968 & 898 & .909 & 0.899 & 0.901 \\
\hline$p$-value of SW & 0.056 & 0.032 & $<0.001$ & $<0.001$ & 0.003 & 0.001 & 0.26 & 0.051 & $<0.001$ & $<0.001$ & $<0.001$ & $<0.001$ \\
\hline Range & 30 & 51 & 13.13 & 13.13 & 62 & 77 & 3.1 & 2.6 & 25 & 25 & 20 & 20 \\
\hline Minimum & 29 & 36 & 25.42 & 26.12 & 88 & 88 & 5.5 & 6.2 & 130 & 130 & 70 & 70 \\
\hline Maximum & 59 & 87 & 38.55 & 39.25 & 150 & 165 & 8.6 & 8.8 & 155 & 155 & 90 & 90 \\
\hline
\end{tabular}

Note: BMI, body mass index; SBP, systolic blood pressure; DBP, diastolic blood pressure; HbA1c, glycated haemoglobin.

Table 2. Microbiome analysis

\begin{tabular}{ccc}
\hline Intestinal Microbiota & Control group & Study group \\
\hline Eschericia coli & $7.391 \pm 2.790 \times 10^{6}$ & $7.468 \pm 2.753 \times 10^{6}$ \\
\hline Proteus spp. & $1.000 \pm 0.000 \times 10^{3}$ & $1.000 \pm 0.000 \times 10^{3}$ \\
\hline Klebsiella spp. & $1.173 \pm 0.978 \times 10^{3}$ & $1.533 \pm 1.833 \times 10^{3}$ \\
\hline Enterobacter spp. & $1.297 \pm 1.021 \times 10^{3}$ & $1.293 \pm 0.161 \times 10^{3}$ \\
\hline Serratia spp. & $1.000 \pm 0.000 \times 10^{3}$ & $1.00 \pm 0.00 \times 10^{3}$ \\
\hline Morganella morganii & $1.000 \pm 0.000 \times 10^{3}$ & $1.000 \pm 0.000 \times 10^{3}$ \\
\hline Citrobactr spp. & $1.000 \pm 0.000 \times 10^{3}$ & $1.081 \pm 0.69 \times 10^{3}$ \\
\hline Pseudomonas spp. & $1.000 \pm 0.000 \times 10^{3}$ & $1.000 \pm 0.000 \times 10^{3}$ \\
\hline Enteroccocus spp. & $2.217 \pm 2.320 \times 10^{6}$ & $3.377 \pm 2.790 \times 10^{6}$ \\
\hline Staphylococcus aureus & $1.000 \pm 0.000 \times 10^{2}$ & $1.000 \pm 0.000 \times 10^{2}$ \\
\hline Akkermansia munciniphila & $4.475 \pm 2.156 \times 10^{9}$ & $2.545 \pm 1.841 \times 10^{9}$ \\
\hline Faecalibacterium prausnitzii & $4.088 \pm 2.302 \times 10^{10}$ & $4.070 \pm 2.388 \times 10^{10}$ \\
\hline Eubacterium spp. & $4.156 \pm 2.358 \times 10^{8}$ & $3.505 \pm 2.298 \times 10^{8}$ \\
\hline Bifidobacterium spp. & $4.251 \pm 2.500 \times 10^{9}$ & $4.452 \pm 2.490 \times 10^{9}$ \\
\hline Bacteroides spp. & $4.324 \pm 2.227 \times 10^{8}$ & $4.529 \pm 2.372 \times 10^{8}$ \\
\hline Raport Firmicutes spp./Bacteroides spp. & $1.128 \pm 1.071 \times 10^{0}$ & $1.124 \pm 1.669 \times 10^{0}$ \\
\hline Candida Albicans & $1.000 \pm 0.00 \times 10^{1}$ & $1.00 \pm 0.000 \times 10^{1}$ \\
\hline Candida Nonalbicans & $1.000 \pm 0.000 \times 10^{1}$ & $1.00 \pm 0.000 \times 10^{1}$ \\
\hline Geotrichum spp. & $1.000 \pm 0.000 \times 10^{2}$ & $1.000 \pm 0.000 \times 10^{2}$ \\
\hline
\end{tabular}

antidiabetics were metformin + glibenclamide combinations (26.70\%), and metformin (12.00\%). The therapeutical administered schemes are presented in Table 5. Also, it was analysed the bacterial species level in the 75 patients from the $\mathrm{SG}$, in the case of treatment presence/ absence. The descriptive analysis is presented in Table 4. For the SG, a Mann Whitney test was applied to find out if patients with treatment have a different gut microbiome structure. Significant differences have been obtained in the case 
Archives of the Balkan Medical Union

Table 3. Bacterial abundance in control group (CG) vs study group (SG)

\begin{tabular}{cccc}
\hline Intestinal Microbiota & CG & SG & $P^{*}$ \\
\hline Escherichia coli & $7.391 \pm 2.790 \times 10^{6}$ & $7.468 \pm 2.753 \times 10^{6}$ & 0.822 \\
\hline Klebsiella spp. & $1.173 \pm 0.978 \times 10^{3}$ & $1.533 \pm 1.833 \times 10^{3}$ & 0.186 \\
\hline Enteroccocus spp. & $2.217 \pm 2.320 \times 10^{6}$ & $3.377 \pm 2.790 \times 10^{6}$ & 0.004 \\
\hline Akkermansia munciniphila & $4.475 \pm 2.156 \times 10^{9}$ & $2.545 \pm 1.841 \times 10^{9}$ & $<.001$ \\
\hline Faecalibacterium prausnitzii & $4.088 \pm 2.302 \times 10^{10}$ & $4.070 \pm 2.388 \times 10^{10}$ & 0.819 \\
\hline Eubacterium spp. & $4.156 \pm 2.358 \times 10^{8}$ & $3.505 \pm 2.298 \times 10^{8}$ & 0.047 \\
\hline Bifidobacterium spp. & $4.251 \pm 2.500 \times 10^{9}$ & $4.452 \pm 2.490 \times 10^{9}$ & 0.584 \\
\hline Bacteroides spp. & $4.324 \pm 2.227 \times 10^{8}$ & $4.529 \pm 2.372 \times 10^{8}$ & 0.560 \\
\hline
\end{tabular}

*Independent Samples Mann-Whitney test
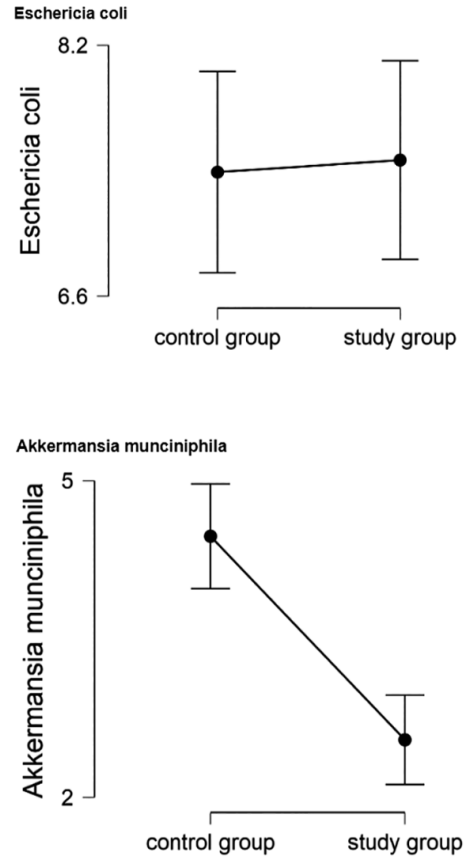

Bifidobacterium spp.
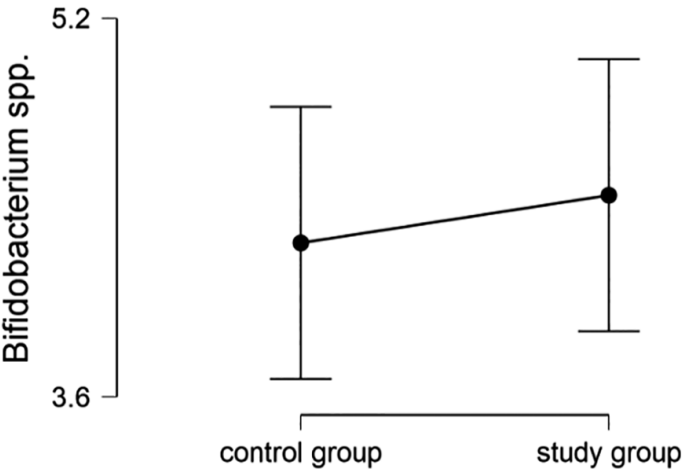
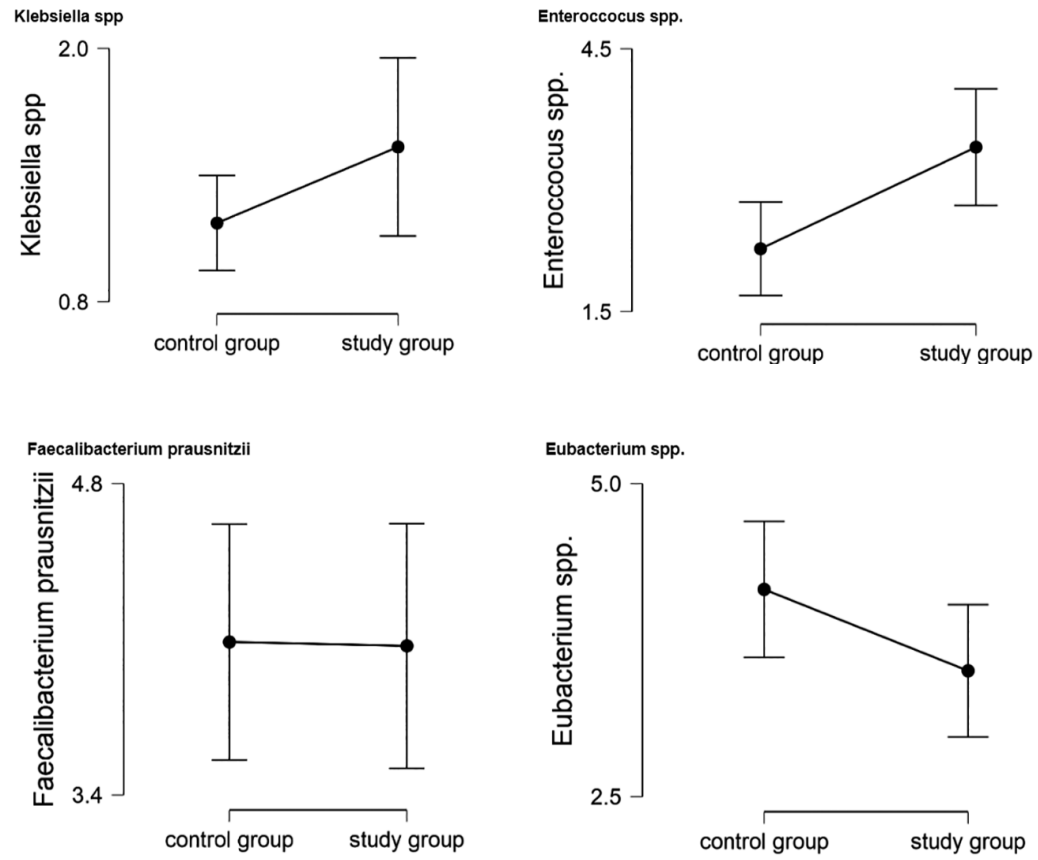

Bacteroides spp.
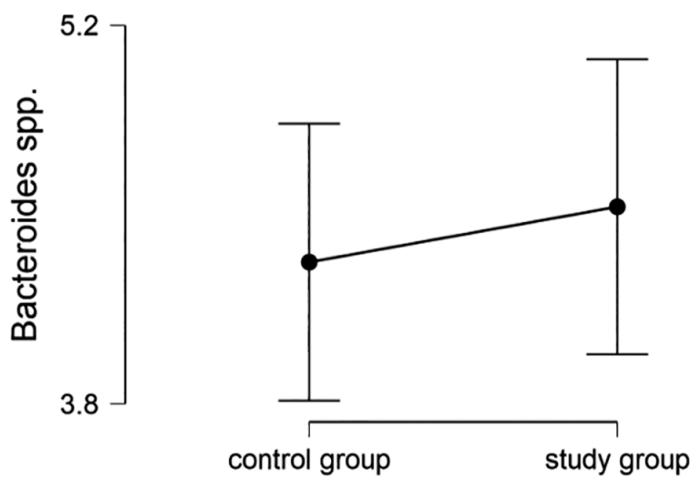

Figure 1. Differences in the number of bacterial species

of A. muciniphila, $\mathrm{p}=0.002$ (Table 5); the patients that followed a treatment have significantly higher values. In all the tested cases, except Faecalibacterium prausnitzii, a growth was registered in the group of patients who had followed a treatment. The entire analysis is detailed in Figure 2.

In the end of the study, a risk analysis has been performed. Considering this cross-sectional study, it 
Table 4. Frequencies of oral antidiabetic treatment

\begin{tabular}{ccc}
\hline Antidiabetic treatment & Frequency & $\%$ \\
\hline Metformin + glibenclamide & 20 & 26.667 \\
\hline Metformin & 9 & 12.000 \\
\hline Gliclazide & 5 & 6.667 \\
\hline Hypoglucidic diet & 41 & 54.667 \\
\hline Total & 75 & 100 \\
\hline
\end{tabular}

Table 5. Bacterial abundance in patients with or without antidiabetic treatment

\begin{tabular}{cccc}
\hline Intestinal Microbiota & Sample 1 & Sample 2 & P* \\
\hline Escherichia coli & $7.253 \pm 2.893 \times 10^{6}$ & $7.661 \pm 2.652 \times 10^{6}$ & 0.517 \\
\hline Klebsiella spp. & $1.000 \pm 0.000 \times 10^{3}$ & $1.976 \pm 2.403 \times 10^{3}$ & - \\
\hline Enteroccocus spp. & $3.377 \pm 2.790 \times 10^{6}$ & $3.259 \pm 2.320 \times 10^{6}$ & 0.624 \\
\hline Akkermansia munciniphila & $2.545 \pm 1.841 \times 10^{9}$ & $4.475 \pm 2.156 \times 10^{9}$ & 0.002 \\
\hline Faecalibacterium prausnitzii & $4.070 \pm 2.388 \times 10^{10}$ & $4.088 \pm 2.302 \times 10^{10}$ & 0.360 \\
\hline Eubacterium spp. & $3.505 \pm 2.298 \times 10^{8}$ & $4.156 \pm 2.358 \times 10^{8}$ & 0.979 \\
\hline Bifidobacterium spp. & $4.452 \pm 2.490 \times 10^{9}$ & $4.251 \pm 2.500 \times 10^{9}$ & 0.624 \\
\hline Bacteroides spp. & $4.529 \pm 2.372 \times 10^{8}$ & $4.324 \pm 2.227 \times 10^{8}$ & 0.624 \\
\hline
\end{tabular}

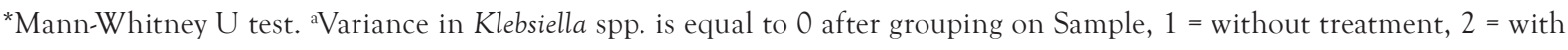
treatment

Table 6. The risk analysis

\begin{tabular}{|c|c|c|c|c|}
\hline \multicolumn{4}{|c|}{ Contingency table } & \multirow{2}{*}{ Results } \\
\hline Akkermansia munciniphila & $\leq 5 \times 10^{9}$ & $>5 \times 10^{9}$ & Total & \\
\hline Without treatment & 38 & 3 & 41 & \multirow{3}{*}{$\begin{array}{c}\mathrm{p}=0.027 \\
\mathrm{OR}=5.227 \\
\mathrm{OR} \in(1.31 ; 21.14)\end{array}$} \\
\hline With treatment & 24 & 10 & 34 & \\
\hline Total & 62 & 13 & 75 & \\
\hline
\end{tabular}

was verified if the lack of treatment can be considered a risk factor of having a lower A. muciniphila quantity. After applying the risk analysis, it resulted that the patients from the group with the disease, who were not under treatment, were significantly more likely to have a lower A. muciniphila quantity $(\mathrm{OR}=5.277,95 \%$ $\in(1.31 ; 21.14), \mathrm{p}=0.027$ ) (Table 6).

\section{Discussion}

More and more data indicate that the development, susceptibility, progression, and severity of T2DM are affected by gut microbiota. Insulin resistance, T2DM, low-grade inflammation and obesity are linked with dysbiosis, altered gut microbiota, that probably indicates a causal role connecting these disorders $^{20}$. Experimental studies and various human trials have shown particular gut bacteria decreased or enriched in T2DM in contrast to healthy controls ${ }^{20}$, and support the connection between gut microbiome and other various MS components ${ }^{21}$.
As the aim of this study was to determine some peculiarities of the composition of the intestinal microbiome in patients with T2DM and MS, our research identified differences in the relative abundance of bacteria in the SG with T2DM and MS. Escherichia coli, Citrobacter spp., and Bacteroides spp. values resulted in being insignificantly higher than in the CG, while the values of Enterococcus spp. were significantly higher; moreover, for Enterobacter spp. and Firmicutes spp./ Bacteroides, the values were insignificantly lower in the SG vs. CG. A. munciniphila and Eubacterium spp. had significantly lower values in the SG compared to the CG.

Generally, compared to healthy subjects (controls), T2DM patients presented increased quantities of branch chain amino acid synthesizing bacteria (Bacteroides vulgatus and Prevotella copri), reduced quantities of opportunistic pathogens (Bacteroides caccae and Clostridium hathewayi) and sulfate-metabolizing bacteria (Desulfovibrio, Lactobacillus gasseri, and Lactobacillus reuteum), and reduced 


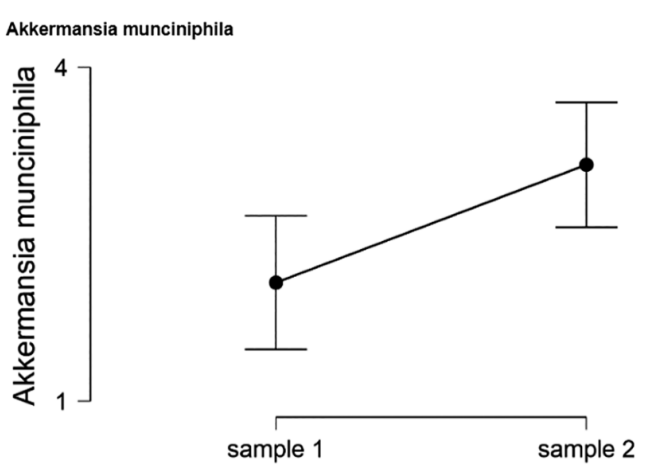

Sample, 1=without treatment , 2=with treatment
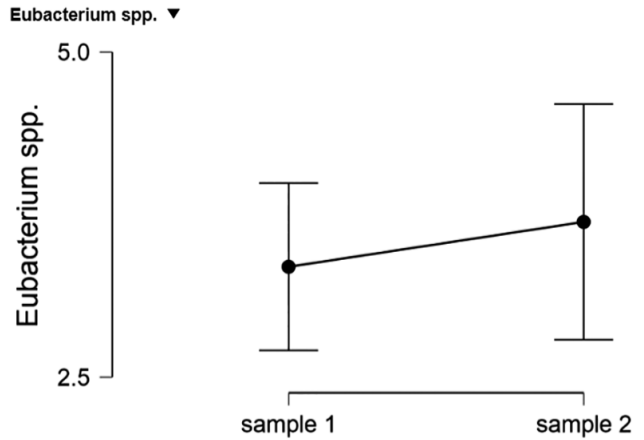

Sample, 1=without treatment , 2=with treatment

Bacteroides spp.

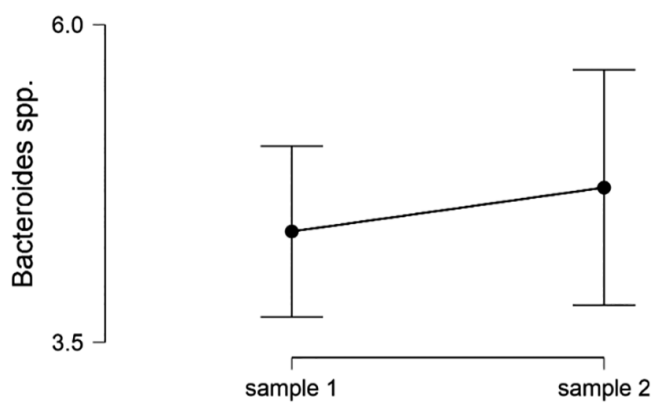

Sample, $1=$ without treatment , 2=with treatment

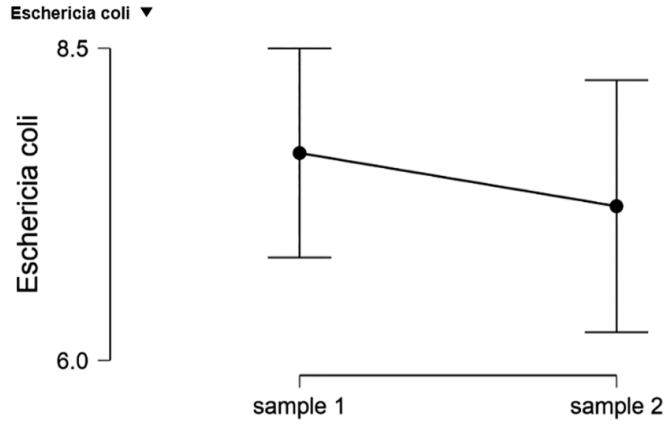

Sample, 1=without treatment , 2=with treatment

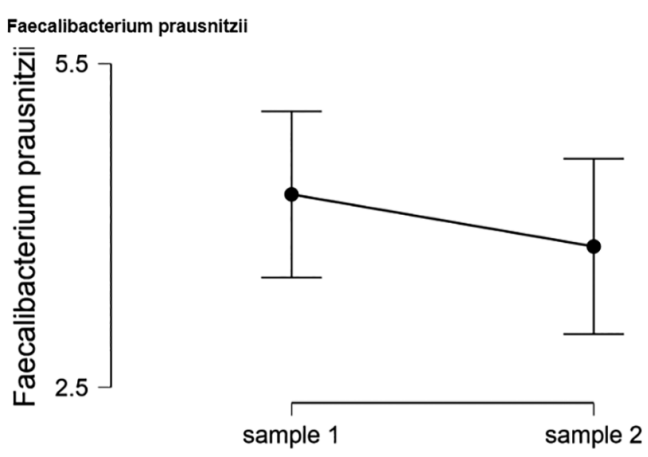

Sample, 1=without treatment , 2=with treatment

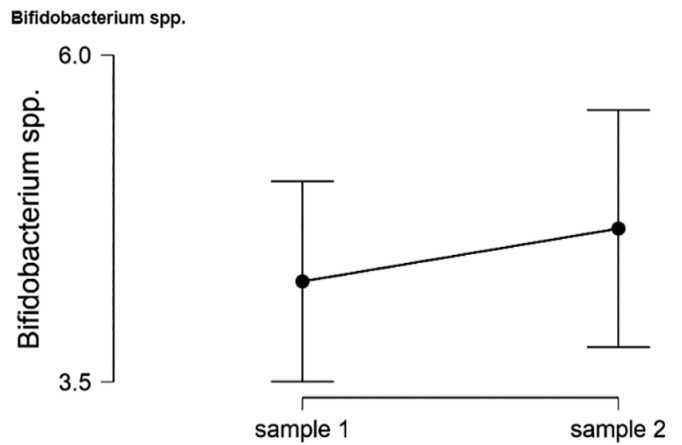

Sample, 1=without treatment , 2=with treatment

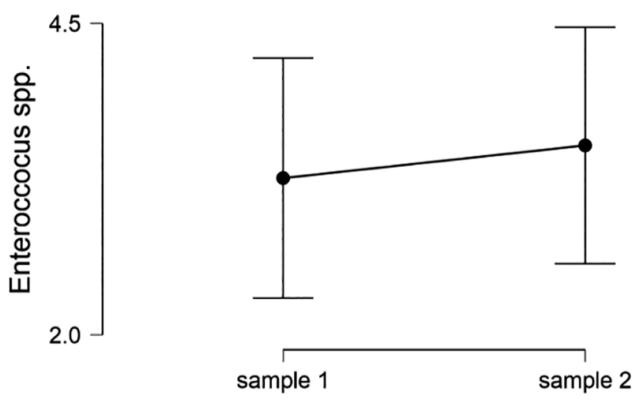

Sample, 1=without treatment , 2=with treatment

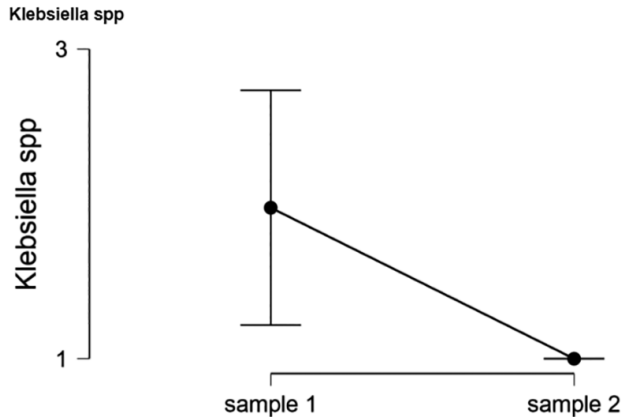

Sample, 1=without treatment , 2=with treatment

Figure 2. Data distribution of the bacterial abundance in patients with or without treatment.

quantities of tryptophan metabolite producing bacteria (Bacteroides, Bifidobacterium) and short-chain fatty acid (SCFA) producing bacteria (Eubacterium rectale, Faecalibacterium prausnitzii, Akkermansia, and Bifidobacterium) $)^{15,22,23}$.
The most indicated beneficial genera in T2DM trials are Bacteroides and Bifidobacterium. Bifidobacterium, a genus comprising microbes that probably offer protection against T2DM, is systematically supported by the literature. Almost all studies indicate a negative 
link between T2DM and this genus ${ }^{24-28}$, though a study indicates opposite outcomes ${ }^{29}$. No significant differences in Bacteroides and Bifidobacterium were reported in the present study between the SG (they resulted in higher amounts in patients treated with oral antidiabetics) and CG.

The host metabolic functions can be influenced by drugs that can alter the gut microbiome (drug microbiome - metabolism axis). Between biguanides, metformin has various effects, it improves glycaemic control and reduces cardiovascular mortality in T2DM patients who are overweight; in newly diagnosed T2DM patients, it is used as first-line treatment and can also prevent $\mathrm{T}^{2} \mathrm{DM}^{30}$. It is also suggested by various evidences that metformin modulates gut microbiota $^{31}$ in high-fat diet (HFD)-fed rodents and humans; additionally, together with high quantities of A. muciniphila, a mucus-degrading gut bacteria, ameliorates glycemia ${ }^{31}$.

The structure of gut microbiota is changed by metformin in both humans and mice, bringing it to a form comparable to that of a healthy host ${ }^{32}$. A decreased quantity of Intestinibacter spp. and Clostridium spp. and a high quantity of Bifidobacterium bifidum, A. muciniphila, Lactobacillus, Escherichia, Shigella spp. are determined by metformin ${ }^{33}$.

As T2DM is a progressive condition, in which higher degrees of hyperglycaemia are depicted, and there is a necessity to increase progressively the dose to preserve the glycemia in normal ranges, combined therapy was applied to target multiple mechanisms ${ }^{34}$. The combinations of metformin and DPP-4 inhibitors, metformin-thiazolidinediones and metformin-sulfonylureas are the most frequently used ${ }^{35}$. Currently, there is limited data on the influence of various combination of therapies on gut microbiome.

In the present study, most patients were administered metformin, alone or in combination with glibenclamide, and the variations in the number of bacteria in the gut microbiome have been determined. Eubacterium, Bifidobacterium, Enterococcus and Bacterioides spp. had an insignificantly higher abundance in the treated group, while the other determined species were insignificantly lower. A. muciniphila was significantly increased in patients with antidiabetic therapy. In recent years, there has been an increase in attention paid to A. muciniphila because of benefits found and proven in reducing body weight, data published in the literature emphasizing the need for colonization with Akkermansia spp. of patients describing metabolic disorders ${ }^{30}$. Both A. muciniphila and mucosal pathology have been modified; moreover, the incidence of inflammatory bowel disease and appendicitis was inversely associated with it ${ }^{36}$, demonstrating the negative association of intestinal A. muciniphila with obesity, diabetes, and other $\mathrm{MS}^{36,37}$. The results of our study suggest that antidiabetic drug treatment combined with diet may significantly influence the intestinal microbiome in patients with diabetes and MS. Several studies to evaluate the response of intestinal microbiota, both to drug and dietary interventions, are needed to pave the way for effective therapeutic approaches in the treatment of T2DM with MS.

\section{Conclusions}

The simultaneous presence of T2DM and MS produces changes in the composition of the intestinal microbiome in the studied patients, leading to a significant increase in the values of Enterococcus spp., and a significant decrease in A. muciniphila and Eubacterium spp. Antidiabetic treatment combined with hypoglycaemic diet positively influence the composition of the intestinal microbiome in patients with both disorders, registering a significant increase in A. muciniphila.

\section{Authors Contribution:}

Conceptualization, R.A.C.A., D.M.T., and C.M.V.; methodology, R.A.C.A. and S.B.; software, R.M.; validation, D.M.T. and S.G.B.; formal analysis, R.M. and S.B.; investigation, R.A.C.A., S.B., and A.G.T; resources, R.A.C.A.; data curation, R.A.C.A. and A.G.T.; writingoriginal draft preparation, R.A.C.A., D.M.T., C.M.V. and S.B.; writing-review and editing, R.A.C.A., D.M.T., R.M, C.M.V. and S.B.; visualization, D.M.T. and S.B.; supervision, S.B.; project administration, S.B. All authors have read and agreed with the final version of this article.

\section{Compliance with Ethics Requirements:}

"The authors declare no conflict of interest regarding this article"

"The authors declare that all the procedures and experiments of this study respect the ethical standards in the Helsinki Declaration of 1975, as revised in 2008(5), as well as the national law. Informed consent was obtained from all the patients included in the study"

"No funding for this study"

\section{Acknowledgements: \\ None}

\section{References}

1. International Diabetes Federation. https://www.diabetesatlas.org/en/. Accessed September 16, 2021.

2. Chatterjee S, Khunti K, Davies MJ. Type 2 diabetes. The lancet. 2017;389(10085):2239-2251. 
3. Chew GT, Gan SK, Watts GF. Revisiting the metabolic syndrome. Medical Journal of Australia. 2006;185(8):445-449.

4. Alberti KG, Eckel RH, Grundy SM, Zimmet PZ, Cleeman JI, Donato KA, Fruchart J-C, James WPT, Loria CM, Smith Jr SC. Harmonizing the metabolic syndrome: a joint interim statement of the international diabetes federation task force on epidemiology and prevention; national heart, lung, and blood institute; American heart association; world heart federation; international atherosclerosis society; and international association for the study of obesity. Circulation. 2009;120(16):1640-1645

5. Kumar S, Behl T, Sachdeva M, Sehgal A, Kumari S, Kumar A, Kaur G, Yadav HN, Bungau S. Implicating the effect of ketogenic diet as a preventive measure to obesity and diabetes mellitus. Life Sci. 2021;264:118661.

6. Guerra JVS, Dias MMG, Brilhante AJVC, Terra MF, García-Arévalo M, Figueira ACM. Multifactorial basis and therapeutic strategies in metabolism-related diseases. Nutrients. 2021;13(8):2830.

7. Huda MN, Kim M, Bennett BJ. Modulating the microbiota as a therapeutic intervention for type 2 diabetes. Frontiers in Endocrinology. 2021;12:153

8. Berg G, Rybakova D, Fischer D, et al. Microbiome definition re-visited: old concepts and new challenges. Microbiome. 2020;8(1):1-22.

9. Savage DC. Microbial ecology of the gastrointestinal tract. Annual Review of Microbiology. 1977;31(1):107-133.

10. Neish AS. Microbes in gastrointestinal health and disease. Gastroenterology. 2009;136(1):65-80.

11. Sekirov I, Russell SL, Antunes LCM, Finlay BB. Gut microbiota in health and disease. Physiological Reviews. 2010.

12. Ley RE, Turnbaugh PJ, Klein S, Gordon JI. Microbial ecology: human gut microbes associated with obesity. Nature. 2006;444(7122):1022-1023.

13. Tilg H, Zmora N, Adolph TE, Elinav E. The intestinal microbiota fuelling metabolic inflammation. Nature Reviews Immunology. 2020;20(1):40-54.

14. Gurung M, Li Z, You H, et al. Role of gut microbiota in type 2 diabetes pathophysiology. EBioMedicine. 2020;51:102590.

15. Qin J, Li Y, Cai Z, et al. A metagenome-wide association study of gut microbiota in type 2 diabetes. Nature. 2012;490(7418):55-60.

16. Ley RE, Bäckhed F, Turnbaugh P, Lozupone CA, Knight RD, Gordon JI. Obesity alters gut microbial ecology. Proc Natl Acad Sci U S A. 2005;102(31):11070-11075.

17. Larsen N, Vogensen FK, van den Berg FW, et al. Gut microbiota in human adults with type 2 diabetes differs from non-diabetic adults. PLoS One. 2010;5(2):e9085.

18. Zymo Research. https://www.zymoresearch.com/collections/zymobiomics-dna-kits. Accessed July 22, 2021.

19. World Medical A. World Medical Association Declaration of Helsinki. Ethical principles for medical research involving human subjects. Bulletin of the World Health Organization. 2001;79(4):373

20. Sircana A, Framarin L, Leone N, et al. Altered gut microbiota in type 2 diabetes: just a coincidence? Curr Diab Rep. 2018;18(10):98

21. Mazidi M, Rezaie P, Kengne AP, Mobarhan MG, Ferns GA. Gut microbiome and metabolic syndrome. Diabetes Metab Syndr. 2016;10(2 Suppl 1):S150-157.
22. Karlsson FH, Tremaroli V, Nookaew I, et al. Gut metagenome in European women with normal, impaired and diabetic glucose control. Nature. 2013;498(7452):99-103.

23. Zhang X, Shen D, Fang Z, et al. Human gut microbiota changes reveal the progression of glucose intolerance. PLoS One. 2013;8(8):e71108.

24. Gao R, Zhu C, Li H, et al. Dysbiosis signatures of gut microbiota along the sequence from healthy, young patients to those with overweight and obesity. Obesity. 2018;26(2):351-361

25. Candela M, Biagi E, Soverini M, et al. Modulation of gut microbiota dysbioses in type 2 diabetic patients by macrobiotic Ma-Pi 2 diet. British Journal of Nutrition. 2016;116(1):80-93.

26. Sedighi M, Razavi S, Navab-Moghadam F, et al. Comparison of gut microbiota in adult patients with type 2 diabetes and healthy individuals. Microbial Pathogenesis. 2017;111:362-369.

27. Wu X, Ma C, Han L, et al. Molecular characterisation of the faecal microbiota in patients with type II diabetes. Current Microbiology. 2010;61(1):69-78.

28. Xu J, Lian F, Zhao L, et al. Structural modulation of gut microbiota during alleviation of type 2 diabetes with a Chinese herbal formula. The ISME Journal. 2015;9(3):552-562.

29. Sasaki M, Ogasawara N, Funaki Y, et al. Transglucosidase improves the gut microbiota profile of type 2 diabetes mellitus patients: a randomized double-blind, placebo-controlled study. BMC Gastroenterology. 2013;13(1):1-7.

30. Corb Aron RA, Abid A, Vesa CM, et al. Recognizing the benefits of pre-/probiotics in metabolic syndrome and type 2 diabetes mellitus considering the influence of akkermansia muciniphila as a key gut bacterium. Microorganisms. 2021;9(3):618.

31. Wu H, Esteve E, Tremaroli V, et al. Metformin alters the gut microbiome of individuals with treatment-naive type 2 diabetes, contributing to the therapeutic effects of the drug. Nature Medicine. 2017;23(7):850-858.

32. Ejtahed H-S, Tito RY, Siadat S-D, et al. Metformin induces weight loss associated with gut microbiota alteration in non-diabetic obese women: a randomized double-blind clinical trial. European Journal of Endocrinology. 2019;180(3):165-176

33. Rosario D, Benfeitas R, Bidkhori G, et al. Understanding the representative gut microbiota dysbiosis in metformin-treated type 2 diabetes patients using genome-scale metabolic modeling. Front Physiol. 2018;9:775.

34. Diaconu C, Nastasa A, Zaki AR, Arsalan M. Type 2 diabetes: a driver for chronic heart failure. The $2^{\text {nd }}$ International Conference on Interdisciplinary Management of Diabetes Mellitus and its Complications - Diabetes mellitus as cardiovascular disease, INTERDIAB 2016 Proceedings, pp. 201-210. Ed. Niculescu. Editors Serafinceanu C, Negoita O, Elian V.

35. Hampp C, Borders-Hemphill V, Moeny DG, Wysowski DK. Use of antidiabetic drugs in the U.S., 2003-2012. Diabetes Care. 2014;37(5):1367-1374.

36. Swidsinski A, Dörffel Y, Loening-Baucke V, et al. Acute appendicitis is characterised by local invasion with Fusobacterium nucleatum/necrophorum. Gut. 2011;60(1):34-40.

37. Diaconu CC. Is there an implication of intestinal microbiota in cardiovascular diseases? Arch Balk Med Union. 2019;54(4):609-611. 